\title{
La pericia técnica como campo en disputa: estrategias de control empresarial en filiales de empresas transnacionales radicadas en Argentina ${ }^{1}$
}

\author{
Technical Expertise as Disputed Field: \\ Strategies for Corporate Control in \\ Argentinian Subsidiaries of Multinational \\ Companies
}

\begin{abstract}
A perícia técnica como campo de disputa: estratégias de controle empresarial nas filiais de empresas transnacionais radicadas na Argentina
\end{abstract}

\section{Claudia Alicia Figari ${ }^{2}$}

Investigadora en el Centro de Estudios e Investigaciones Laborales (CEIL) del Consejo Nacional de Investigaciones Científicas y Técnicas (Conicet), Buenos Aires, Argentina

cfigari@ceil-conicet.gob.ar

Recibido: $17 / 10 / 2014$

Aprobado: 24/11/2015

1 El presente artículo se enmarca en proyectos financiados por la Agencia Nacional de Promoción Científica y Tecnológica, el Consejo Nacional de Investigaciones Científicas y Técnicas, y la Universidad de Buenos Aires.

2 Magíster en Ciencias Sociales del Trabajo. Doctora con orientación en Ciencias de la Educación. 


\title{
Resumen
}

Este artículo problematiza los saberes requeridos y las clasificaciones atribuidas a los operarios a partir de las estrategias corporativas de control social. Se sostiene que la colaboración en la mejora continua y la intensificación en los ritmos de trabajo constituyen caras de una misma moneda y posibilitan la apropiación empresarial de la pericia técnica obrera a través de las "células de trabajo". Las estrategias empresariales dejan al descubierto fisuras que, a veces, se traducen en resistencia de los trabajadores. Exponemos hallazgos derivados de estudios de caso en empresas del sector neumático y automotor. La estrategia metodológica es cualitativa, centrada en entrevistas a operarios y delegados sindicales. También se han analizado informes de sostenibilidad y convenios colectivos de trabajo.

Palabras clave: gestión de personal; cualificación profesional; relaciones laborales; negociación colectiva; desarrollo de la carrera.

\begin{abstract}
This paper studies the required knowledge and professional hierarchies assigned to the workers through corporate strategies of social control. It is argued that collaboration on "continuous improvement" and intensification of working pace allow corporate appropriation of technical expertise through "working cells". Corporate strategies unveil fissures that sometimes turn into workers' resistance. We present findings from case studies in tire and automotive sectors. The methodological strategy is qualitative, focused on workers and trade union delegates interviews; sustainability reports and collective labor agreements are also analyzed.
\end{abstract}

Keywords: human resources management; professional qualification; labor relations; industrial relations; collective bargaining; career development.

\section{Resumo}

Este artigo problematiza os saberes exigidos e as classificações atribuídas aos operários, a partir das estratégias corporativas de controle social. Sustenta-se que a colaboração na melhora contínua e a intensificação nos ritmos de trabalho, constituem caras de uma mesma moeda e possibilitam a apropriação empresarial da perícia técnica operária, através das "células de trabalho". As estratégias empresariais deixam ao descoberto fissuras que, às vezes, se traduzem em resistência dos trabalhadores. Expomos resultados derivados de estudos de caso em empresas do sector pneumático e automotor. A estratégia metodológica é qualitativa, centrada em entrevistas a operários e representantes sindicais e em revisão de informes de sustentabilidade e convênios coletivos de trabalho.

Palavras-chave: gestão empresarial; qualificação profissional; relações de trabalho; negociação coletiva; desenvolvimento da carreira.

\footnotetext{
¿Cómo citar este artículo? / How to quote this article?

Figari, Claudia. «La pericia técnica como campo en disputa: estrategias de control empresarial en filiales de empresas transnacionales radicadas en Argentina». Sociedad y economía, No. 30 (enero - junio de 2016): 199-223.
} 


\section{Introducción}

Los procesos de modernización empresarial han modelado un nuevo orden productivo, laboral y profesional en los últimos veinte años. Las líneas de investigación desarrolladas han aportado hallazgos contundentes con relación a las consecuencias de las políticas empresariales en el requerimiento y valorización de los saberes que movilizan los trabajadores en el acto de trabajo y en la necesidad de inscribir lo anterior en el conflicto entre el capital y el trabajo. Los reglamentos corporativos se imponen unilateralmente, movilizando, en los hechos, nuevas posiciones que imprimen fragmentación en los colectivos obreros y se constituyen en una vía para la variabilidad salarial. De esta manera, las lógicas individualizantes se expresan en la gestión por competencias y de las clasificaciones profesionales (Monchatre 2009), así como en la centralidad de trayectorias individuales, donde la competencia entre trabajadores, la meritocracia y la colaboración son especialmente valorizadas (Jonnaert et al. 2008; Muñiz 2009; Figari 2011a). Estas lógicas cobran predominancia en las grandes empresas y se encuentran estrechamente vinculadas con los sentidos neoliberales y toyotistas. Estos sentidos cobran presencia en los contenidos específicos que se definen para la gestión del trabajo (Roper, Ganesh e Inkson 2010; Lima 2010; Delfini 2011) y se expresan también en la negociación colectiva. En el contexto que se viene describiendo, los operarios han sido especialmente afectados a partir de las políticas de modernización empresarial. Mientras que en los años de 1990 las fuentes de exclusión se multiplicaron, posteriormente la precarización (vía la tercerización) y la selectividad (por ejemplo, para la movilidad profesional y el acceso a puestos de mando) han sido tendencias recurrentes y han dado fisonomía al orden laboral y profesional (Antunes 2009; Ollivier 2011). Este artículo se focaliza en el requerimiento de saberes y en su valorización a través de las clasificaciones profesionales en los niveles inferiores del mando, en especial en las categorías operarias. Del orden hegemónico empresarial se derivan múltiples exigencias corporativas que se traducen en una intensificación del trabajo, en la búsqueda de colaboración y en límites para la movilidad profesional. Detrás se dirime la desvalorización del saber hacer en las clasificaciones, si bien esos saberes resultan indispensables para el funcionamiento productivo de las empresas. En ese contexto, se moviliza una gestión del conocimiento empresarial basada en la demanda de competencias reguladoras (Bernstein 1998) y en evaluaciones del desempeño de los trabajadores - que alcanzan en la actualidad los niveles inferiores del mando- .

Este artículo expone hallazgos de la investigación realizada en el segundo lustro de los años de 1990 y más recientemente en el último lustro en grandes empresas del sector del neumático y automotor respectivamente. Se ponen en evidencia tendencias que, impulsadas desde los años de 1990, se consolidaron en la pasada década y permiten problematizar los saberes requeridos y las clasificaciones atribuidas a los operarios a partir de las exigencias corporativas de colaboración y competencia hacia los trabajadores. Especialmente, las lógicas corporativas que fomentan el trabajo en grupo (Datchary 2008) son puestas de manifiesto como espacios que posibilitan la apropiación de los saberes técnicos en función de las exigencias corporativas. Esta vía de análisis conduce también 
a problematizar en qué medida la gestión por competencias, las evaluaciones de desempeño y las clasificaciones profesionales asociadas con las matrices corporativas cobran presencia en los convenios colectivos de trabajo.

Los estudios realizados en los años de 1990 corresponden a grandes empresas, filiales argentinas de holdings transnacionales que pertenecen a la industria del neumático (Angélico y Figari 1999). Se trata de organizaciones que han desarrollado una estrategia de modernización productiva, con base en las políticas de mejora continua de la calidad y en la implementación de cláusulas emblemáticas flexibilizadoras como es el trabajo por turnos continuo, materia que se instrumenta a través de actas de acuerdo en las cuatro empresas hegemónicas del sector del neumático en ese período. Se desarrolló una aproximación cualitativa, centrada fundamentalmente en la administración de entrevistas a delegados sindicales del Sindicato Único de Trabajadores de la Actividad del Neumático (SUTNA) y del Sindicato de Empleados y Personal Jerárquico de la Actividad del Neumático Argentina (SEPJANA) conformado a mediados de los años de 1990. A su vez, fueron analizados diversos convenios colectivos de trabajo (CCT) y actas de acuerdo; también se profundizó en una gran variedad de material corporativo empleado para la capacitación en los nuevos sistemas de trabajo, sustentados en las políticas de mejora continua de la calidad.

Los estudios realizados en el último lustro corresponden a una planta automotriz localizada en Zárate, provincia de Buenos Aires, con casa matriz en Estados Unidos. Las principales fuentes analizadas corresponden a entrevistas colectivas a jóvenes operarios -técnicos-, a convenios colectivos de trabajo, material corporativo empleado en la formación de trabajadores e informes de sustentabilidad.

El recorrido realizado en esta investigación aporta elementos sustanciales, ya que pone de manifiesto puntos de inflexión en la lógica de expansión del capital y en la especialización de herramientas corporativas que tienen implicancias directas en el requerimiento y valorización de los saberes productivos. Justamente, el caso del sector del neumático es anticipatorio de las técnicas gerenciales que luego tendrán desarrollo y articulación sistémica en la última década según hemos constatado en nuestras investigaciones más recientes.

Las tradiciones investigativas en la década de 1990, que llevaron a situar la mirada en las formas que asumía la denominada modernización, se complementaron con investigaciones posteriores que ponen en evidencia la matriz conceptual de las doctrinas gerenciales del capitalismo global. Esa matriz se expone claramente en la filial argentina de la empresa Toyota que estudiamos. Un elemento a destacar es que la empresa automotriz se instala en Zárate, provincia de Buenos Aires, en 1996, período en que se desarrolla la disputa obrero-patronal en el sector del neumático, cuando una de las empresas impone modificar la organización del tiempo de trabajo y así impulsa, por medio de la negociación, el trabajo por turnos continuo, materia expresiva de la flexibilización laboral ${ }^{3}$.

3 Se trata de una modalidad de trabajo que implica rotar, por ejemplo, cada semana sucesivamente trabajando mañana, tarde y noche. La continuidad incluye un esquema organizativo en el cual los trabajadores se desempeñan, en el caso investigado, durante seis días consecutivos y descansan dos. 
En ese marco se ponen de manifiesto los límites que encuentra la empresa en algunos sectores de trabajo relacionados con la experiencia acumulada por parte de los trabajadores para realizar funciones técnicas que exigen movilizar saberes técnicos complejos.

En la terminal automotriz estudiada, el denominado Toyota Production System (TPS) expresa la base conceptual fundamental de las lógicas corporativas que se van implementando en grandes empresas transnacionales, sin importar el sector económico de que se trate.

Este artículo está estructurado en tres secciones: en la primera se aporta al debate conceptual con relación a la disputa de saberes y configuraciones profesionales en los espacios de trabajo. En la segunda se enfatiza en las tendencias predominantes de las políticas gerenciales desde los años de 1990 y se analiza la expresión que han cobrado en las empresas estudiadas; en ese marco, el propósito es problematizar la disputa en torno a los saberes productivos y a las herramientas de gestión empresarial empleadas con el fin de cooptar la pericia técnica. El tercer apartado y como derivación de los anteriores, se focaliza en las implicancias que traen aparejadas las doctrinas corporativas flexibilizadoras y de la mejora continua en las clasificaciones profesionales. Asimismo, se identifican diferentes situaciones en las cuales se expresan las acciones de resistencia de los trabajadores.

\section{Saberes y clasificaciones: requerimientos y valorizaciones}

En el marco del debate calificaciones-competencias, se ha destacado la centralidad del saber ser en contextos de modernización empresarial (Dombois 1994; Tanguy 2003). Sin embargo, en menor medida se ha analizado su vínculo orgánico con la matriz contemporánea de disciplinamiento laboral y cultural. En esta línea se señalan los aportes de Batista (2006), quien se refiere a "saberes gerenciales" para connotar el espacio de imposición de sentidos gerenciales tributario de la mejora continua. Al respecto, en otro trabajo desarrollé la noción de saberes corporativos (Figari 20llb), para aludir a aquellos conocimientos que subyacen en las doctrinas corporativas y requieren ser transpuestos en el cotidiano de trabajo. Justamente, la centralidad que asume la actividad cognitiva en las doctrinas toyotistas contrasta, tal como se analiza en este artículo, con la subalternización de la experiencia acumulada por los trabajadores. Más allá de las contradicciones en la semántica toyotista, el lenguaje corporativo se extiende fronteras dentro y más allá del suelo de las fábricas (Quintana 2009; Giniger 2011).

La lucha de sentidos en el corazón de la acción hegemónica desnuda hoy, como ayer, la disputa de saberes (empresarios, obreros) y de jerarquías y clasificaciones, tributaria de las posiciones de dominio del capital en un esquema territorializado en las empresas ${ }^{4}$. Las doctrinas corporativas - procedentes de

4 Me refiero a sistemas corporativos para caracterizar la fisonomía local (en el ámbito de una planta, filial de una empresa transnacional) que adquieren las doctrinas corporativas definidas en las casas matrices, mediadas por la legislación laboral local, la historia de lucha y conflictividad obrero-patronal. 
casas matrices - se especializan en contextos situados, a partir de sus rediseños se articulan varias herramientas corporativas y adquieren protagonismo los dispositivos de control social y profesional. La praxis empresarial cobra especial relevancia y se destaca el esfuerzo sistemático de la dirigencia para transmitir eficazmente los "mensajes corporativos" en los espacios de trabajo. En este proceso resulta indispensable especializar la doctrina corporativa teniendo en cuenta las exigencias técnicas y productivas de cada sector de la producción. En este marco, los saberes y las clasificaciones debieran ser analizados en sus vínculos fundamentales. La investigación realizada ha profundizado en la disputa de poder y sentidos que gobierna en las organizaciones empresariales. Estas aproximaciones ahondan en el vínculo pedagógico inherente a las relaciones sociales de producción (Neves 2005; Kuenzer 2011). Es en este sentido que se hace referencia a la importancia de desarrollar una pedagogía crítica del trabajo que ponga en evidencia y someta a la crítica la praxis empresarial y la desplegada por los trabajadores.

En este contexto, el enfoque que sostenemos profundiza en las relaciones saber/poder y en especial en los mecanismos complejos empleados para configurar y sostener el orden empresarial. De esta forma, el orden profesional encuentra un claro sustento en los criterios de normatividad imperantes - basados en la mejora continua como principio rector- que se expresan, desde nuestra tesis, en la naturaleza y alcance de los saberes requeridos, valorizados y en la disputa por las clasificaciones profesionales. Una lectura dinámica de la organización supone captar las configuraciones profesionales (Rozemblatt 1999). La noción de configuración, desarrollada por Norbert Elias (1993), permite objetivar las interrelaciones entre condiciones subjetivas y objetivas, o bien entre sujetos y organización. Específicamente, los saberes movilizados por los trabajadores en el acto de trabajo se despliegan en una estructura posicional, jerárquica, donde los saberes y los contenidos técnicos de los puestos/funciones se traducen en un orden clasificatorio y de esta forma en un sistema de remuneración. La configuración teje la trama (no visible) entre el orden estructural y los procesos de subjetivación; ambos dominios resultan dos caras de la misma moneda, que Elias expone profusamente al referirse a la dialéctica entre control y autocoacciones. Esta noción se hace potente al abrir un camino para conectar conceptual y empíricamente los itinerarios/recorridos que las organizaciones habilitan y las trayectorias profesionales que los sujetos trabajadores transitan. El estudio de los saberes requeridos y valorizados fue considerado marginalmente en su vínculo orgánico con la estructura posicional y en general con las clasificaciones profesionales. Al respecto, los aportes de Friedmann y Naville (1971) fueron contundentes al postular la necesidad de ahondar en la división social del trabajo. Es desde allí que la disputa de saberes y posiciones cobra sentido al avanzar en la comprensión de las relaciones entre calificaciones y clasificaciones profesionales y para problematizar las posibilidades y restricciones que se les presentan, por ejemplo, a los operarios para la movilidad profesional.

Desde las tesis que se sostienen, las reconfiguraciones del orden laboral y profesional derivadas de los sistemas de mejora continua ponen en evidencia profundas interrelaciones entre normalizaciones y selectividades que restringen, tensionan, imponen claros techos a las movilidades profesionales y asocian la 
posibilidad de ascenso con un comportamiento laboral que demuestre adhesión a las exigencias corporativas. Es decir, la colaboración de todos (con el proyecto de la firma) se expresa, a la vez, en las posibilidades para algunos trabajadores de transitar un itinerario profesional, que se traduzca en movilidad profesional ascendente ${ }^{5}$. El disciplinamiento en el trabajo expresa, así, las exigencias normativas/reguladoras derivadas del conocimiento oficial empresarial ${ }^{6}$, cuestión que no puede soslayarse en un examen detenido del espacio profesional, que se hace mucho más selectivo y excluyente. Desde esta perspectiva, es en el marco de la disputa hegemónica que se dirimen también los requerimientos y valorizaciones de los saberes técnicos-productivos de la fuerza laboral, indispensables para la realización del proceso de trabajo que se despliega en contextos situados. Me refiero a aquellos saberes directamente relacionados con las exigencias técnicas y productivas del proceso de trabajo, fruto de la experiencia acumulada y de la formación inicial. Las prácticas que despliegan los trabajadores no pueden ser deslindadas del esfuerzo patronal por imponer y regular eficazmente determinados procesos de subjetivación en el trabajo (Montes 2005). Es decir, el grado de coherencia de prácticas y significaciones (León 2009), en tanto lógicas de acción y de sentido, no significa que no respondan a matrices disciplinarias reificadas. El debate histórico, y por cierto actual, en los estudios que analizan las relaciones entre control patronal y resistencia, a veces, ha sido subalternizado al no enfocar las formas específicas en que el capital ejerce su domino sobre los trabajadores. Es en este marco que la mirada hacia los trabajadores resulta potente, si bien discutimos con aquellos enfoques que la descentran del problema del poder y en definitiva de la disputa obrero-patronal. Se trata de una vieja y recurrente, pero actualizada, polémica en los estudios sociales del trabajo. De igual forma el problema del saber, de la formación y de las clasificaciones ofrece aún un panorama a ser explorado con mayor profundidad en el marco de las actualizaciones del capitalismo global. Los saberes requeridos para determinados puestos técnicos de trabajo a veces no resultan valorizados ni traducidos en claros criterios para las movilidades profesionales. Sin embargo, los trabajadores son cada vez más exigidos en la movilización de saberes más complejos, aportan mejoras al proceso que resuelven en tiempo real al poner en juego de saberes implícitos difíciles de ser codificados. Si bien el lugar de trabajo se constituye en un espacio donde se crean, recrean, transmiten y distribuyen saberes, la disputa del capital se expone en forma permanente al generar, como se demuestra en este artículo, instancias de apropiación de saberes técnicos que nutren a los sistemas corporativos y a la posibilidad de funcionamiento de las empresas. Esta apropiación no supone desaparición de la masa crítica de saberes que portan los

5 Los aportes de Bourdieu (1984) han sido significativos al visibilizar las conexiones entre las estructuras posicionales, los saberes en disputa y los procesos de subjetivación. La dinámica operante entre normalizaciones y distinciones simbólicas -en el lenguaje bourdiano- asume implicancias fundamentales en las movilidades profesionales. Así, se destaca, por ejemplo, el peso gravitatorio que asumen las adhesiones a las doctrinas corporativas por parte de los trabajadores.

6 Saberes expertos del management, sistematizados y configurados en reglas y principios corporativos. Así, la codificación del conocimiento experto aporta la función legitimadora de las doctrinas corporativas. 
trabajadores, dado que la pericia técnica se recrea en forma constante, es justamente allí donde radica el poder obrero. Esta ha sido una vieja batalla por parte de los sindicatos al negociar colectivamente los criterios para la valorización de saberes y su traducción en los sistemas de clasificación profesional. En la historia más reciente y frente a las transformaciones del capitalismo global, los límites para los trabajadores en los horizontes de movilidad se ven especialmente restringidos. Además, cobran centralidad las lógicas individualizantes asociadas con la gestión de las competencias y de los desempeños que gobiernan en escenarios en los cuales se definen, en los hechos, acuerdos individuales para el desarrollo de las carreras. En consecuencia, el estudio de los saberes que se les demandan a los trabajadores no puede disociarse de la polémica en cuanto a la puesta en valor del trabajo (Lichtenberger 2000) y del reconocimiento de la pericia técnica. Sin embargo, el movimiento del capital y las estrategias corporativas empresariales consolidadas en los últimos años definen un escenario cada más incierto: aun requiriendo de esa pericia la retribuye diferencialmente según sea el grado de adhesión a los principios corporativos imperantes.

\section{Intensificación del trabajo y colaboración con la firma: tensiones persistentes en los grupos de trabajo}

La doctrina gerencial contemporánea articula complejamente formas de intensificación del trabajo con tecnologías que invocan la participación, la autonomía y el involucramiento. Ambas estrategias se sustentan mutuamente y desde esta tesis es posible visibilizar los mecanismos sofisticados que operan sobre la fuerza laboral para lograr consentimiento (Soich 2008; Giniger 2012).

Para los operarios, la estrategia empresarial se expresa a la vez (y controversialmente) en precarización de las condiciones de empleo, de trabajo y en la sujeción de los trabajadores a un universo discursivo que los sitúa, antes que nada, como "colaboradores".

En los años de 1990 se instrumentó, como parte de las políticas empresariales modernizantes, un fuerte recambio en la composición de la fuerza laboral y una nueva gestión del trabajo basada en la doctrina flexibilizadora que fragilizaba el vínculo laboral y establecía formas de cooperación con el fin de aumentar la productividad. En ese contexto, las doctrinas gerenciales fortalecían una gran variedad de herramientas de gestión e instalaban lógicas individualizantes y competitivas entre los trabajadores. La gestión del conocimiento oficial empresarial, de las clasificaciones y la política de variabilidad salarial se sustentaron en modificaciones que, de a poco, fueron definiendo nuevos criterios en los reclutamientos y en las movilidades. Ya en dicha década la semántica del management se instala y cuenta cada vez con mayor difusión a través, incluso, de los medios de prensa, las consultoras, las fundaciones, etc. (Alonso y Fernández 2011; 2013). Sin embargo, el esfuerzo comunicacional distaba de afincar en el cotidiano laboral y de articularse con otras herramientas corporativas. Esta cuestión se irá efectivizando y posibilitará la consolidación del accionar hegemónico empresarial en el capitalismo global (Figari 2013). En la primera década del milenio, se articulan herramientas de variada naturaleza que se extienden a la sociedad civil y política, a través, por ejemplo, de los programas de responsabilidad social 
empresarial (Delgado 2007; Graciolli y Lamana 2010), los programas de formación y certificación de competencias (Riquelme y Herder 2007), las universidades corporativas e, incluso, algunas universidades públicas y privadas, como espacios sensibles en la formación de los cuadros dirigenciales empresariales.

La estrategia empresarial fortalecerá también la herramienta de la tercerización, que se constituirá en una vía central para el reclutamiento de la fuerza laboral. Así, las formas precarias se sustentan en las doctrinas gerenciales contemporáneas (Edwards y Collinson 2002; Stewart et al. 2005) que afinan sus instrumentos de dominio sobre la fuerza laboral con el propósito de formar nuevas subjetividades (Alves 2006). La lucha hegemónica se traduce en disputa de saberes y clasificaciones y expone particularmente la batalla cultural y política por la conciencia obrera (Lukács 1985; Mészáros 2004).

La coexistencia de las doctrinas gerenciales contemporáneas, basadas en el denominado management participativo y en la flexibilidad laboral, da apertura a fisuras en la apuesta empresarial por consolidar la hegemonía sobre la fuerza de trabajo. Y, justamente, esas fisuras abren paso a múltiples acciones de resistencia entre los trabajadores. En los grupos de trabajo-espacios instrumentados desde la estrategia patronal y constituidos en ámbitos desde los cuales se imponen los sentidos oficiales empresariales-, se expresa ese tensionamiento, allí se exacerban y multiplican las "distinciones simbólicas" entre los trabajadores (algunos serán líderes, facilitadores, integrantes de grupos de mejora continua), y se habilita una vía para la diferenciación salarial. Desde nuestra tesis, los grupos se constituyen en un espacio de apropiación empresarial de los saberes técnicos obreros en el marco del requerimiento corporativo de disponibilidad plena de los trabajadores hacia los principios que reglan el orden empresarial. Allí, coexisten trabajadores efectivos y tercerizados - que realizan las mismas funciones- y se intensifica el trabajo humano.

Los hallazgos que se presentan a continuación corresponden a dos casos considerados paradigmáticos de la tesis que sostiene las continuidades y articulaciones entre la doctrina flexibilizadora y el paradigma modernizante de la colaboración en la mejora continua. Asimismo, presentan aportes nodales para problematizar cómo son afectados los trabajadores operarios a quienes se les exige, en un marco de intensificación del trabajo, colaboración y aportar los saberes productivos en forma sistemática en los grupos de trabajo.

En el segundo lustro de la década de 1990 se impone en el sector del neumático, a través de la negociación colectiva, una materia emblemática de la flexibilidad laboral que expresa sensiblemente las estrategias de intensificación de la fuerza laboral: el trabajo por turnos continuo, cuya regulación, por medio de actas de acuerdo en el ámbito de las empresas, significaba incorporar un cuarto turno de operarios a tiempo parcial, y a los efectos de reemplazar a los trabajadores que se encontraban de franco, es decir, en su día de descanso. Estas cláusulas reguladas colectivamente se articulaban con un discurso modernizante que invocaba el cambio cultural en pos de la mejora continua de la calidad.

El caso de la terminal automotriz que se instala en Zárate expone el sentido más emblemático del principio rector de la mejora continua en los grupos de trabajo. A diferencia de las empresas del neumático, en la terminal automotriz 
todos los operarios son jóvenes técnicos ${ }^{7}$-tendencia que, de a poco, se irá extendiendo en las grandes empresas-. En ambos casos, el aporte de los saberes productivos (que es apropiado por la patronal, pero también resistido por los trabajadores) abona al principio de la mejora continua en un contexto de intensificación del trabajo. También, en ambos casos, la negociación colectiva se constituye en un vehículo para imponer los sentidos oficiales empresariales.

\subsection{El trabajo continuo para la mejora continua}

En la empresa de neumáticos se expone expresivamente la intensificación del trabajo y a la vez la búsqueda de colaboración. El estudio realizado se constituyó en una vía fértil para visibilizar los vasos comunicantes (a veces disociados en los discursos oficiales) entre las técnicas toyotistas -asociadas a la mejora continua y a las competencias- y las formas de intensificación del trabajo humano, en pos de la economía de tiempo. La estrategia empresarial combinaba un discurso modernizante, ligado al cambio cultural (tendiente al recambio de la fuerza laboral), y la promoción generalizada (si bien en los hechos se instrumenta gradualmente) de una organización del tiempo de trabajo basada en el trabajo por turnos continuo. En ese contexto, la empresa impulsa la reducción de los planteles que afectará a las categorías operarias y a las de supervisión (muchos trabajadores idóneos que por carrera interna habían alcanzado esa primera categoría de mando). Esta situación influyó particularmente en el sindicato, por la pérdida de afiliados. Es en este marco que se constituye el Sindicato de Empleados y Personal Jerárquico de la Actividad del Neumático Argentina (Sepjana), que incluirá ya no solo a los supervisores:
A finales de los ochenta eran 2.500 personas empleadas, ahora somos aprox. 650
(...) con excepción de algunos sectores no existió informatización de algún proceso o introducción de microelectrónica; y teniendo en cuenta que con una producción de 7.200 cubiertas se pasa a una de 8.200 con menor cantidad de operarios, queda de manifiesto que se produce un fuerte aumento en la carga de trabajo (...) también entre los signos de la reestructuración subyace la idea de homogeneizar a todos los trabajadores en torno de lo que puede ser "una gran familia", y la empresa intenta negociaciones de manera individual con los empleados: si no entras en el perfil, no entras en la reestructuración (...) como corolario, la empresa logró un cambio muy importante en el sistema de trabajo al pasar de un sistema rotativo con descansos sábados y domingos a formas continuas de trabajo (dirigente sindical del Sepjana, desempeñaba funciones en la empresa localizada en Hurlingham).

En la década de 1990, los debates en el campo de las Ciencias Sociales del Trabajo ahondaban en el estatus de la modernización, y hubo cierto consenso en que las transformaciones se relacionaban más con tecnologías sociales que con la introducción de cambios sustanciales en el nivel de las tecnologías de base microelectrónica e informática. Si bien se podían observar situaciones de implementación de estos sistemas en sectores específicos, coincidimos con aportes de algunas investigaciones que postulaban que más que cambiar al trabajo, el

7 Operarios que cuentan con la credencial educativa que corresponde al nivel medio técnico. 
propósito empresarial era cambiar a los trabajadores (Linhart 1997). Como antesala de los cambios, los despidos se multiplicaban, o bien hacía estragos el menú de posibilidades que la reforma laboral en Argentina ofrecía a la clase empresarial: las contrataciones a término, el aumento de tareas, la movilidad geográfica y la intensificación de los ritmos de trabajo. Para quienes conservaban los empleos, se instalaba como palabra clave en la voz del management la idea de cambio cultural, asociada a una clara disputa ideológica empresarial, que aún no encontraba instrumentos precisos para una plena implementación en los espacios de trabajo. En el sector del neumático, 1996 y 1997 fueron años claves por el impulso dado por la patronal para implementar el trabajo por turnos continuo. Esta materia se pretendía instalar por medio de la negociación colectiva. Como en otras empresas, en especial aquellas privatizadas, la negociación instrumentó cláusulas emblemáticas de la flexibilidad (como por ejemplo firmar aspectos concernientes a la polivalencia funcional y a los acuerdos por productividad), fundamentalmente a través de actas de acuerdo en el ámbito de las empresas.

La negociación del trabajo por turnos continuos y de la productividad se entremezcla con el discurso gerencial que pide colaboración y de esta forma aporta a un proyecto común sobre la base de impulsar un cambio cultural, a través de la participación en la mejora continua. Así, la mejora continua se expresa en la pretensión empresarial de imponer una organización del tiempo de trabajo por turnos rotativos y continuos, a través de la negociación colectiva, como medio para vehiculizar las cláusulas de flexibilidad laboral. Los trabajadores pasarían de un sistema con descanso dominical a otro de seis días de trabajo y dos de descanso en cualquier día de la semana, además de dejar de percibir a un $200 \%$ el pago de horas trabajadas en sábado y domingo. La empresa incorporaba un cuarto turno para cubrir los días de descanso y vacaciones, cuestión que saldrá a respaldar la autoridad administrativa, a partir del requerimiento empresarial de intervenir en el conflicto frente al rechazo inicial a negociar por parte del sindicato. La estrategia flexibilizadora de la empresa pretendía instalar un régimen vacacional móvil y distribuía las vacaciones a lo largo del año. El funcionamiento continuo de la empresa (que pasa de trabajar 276 días a 360 días al año) operaba también sobre los cuerpos al consolidar un trabajo continuo que era medido en forma continua según su productividad. La negociación del trabajo por turnos continuos se regula finalmente por actas de acuerdo en las cuatro empresas que concentraban la actividad del neumático en los años de 1990. La imposición empresarial supuso despidos de trabajadores (al no aceptar el sindicato la negociación inicialmente) y la intervención de la autoridad administrativa legitimó el accionar patronal. Así, la doctrina flexibilizadora entramada con las lógicas modernizantes toyotistas fue vehiculizada por imposición de la empresa a través de la negociación colectiva $^{8}$ (Marshall y Perelman 2004).

8 La negociación incluyó múltiples procesos articulados de conflicto, negociación, acuerdos en una relación de fuerza desfavorable al sector del trabajo. Allí se expresa la interrelación entre un accionar instituyente e instituido, que es dinámico y conflictual. Las disputas entre el capital y el trabajo objetivan esa interrelación en regulaciones establecidas en convenios colectivos de trabajo o en actas de acuerdo. 
En el marco descrito, el departamento de capacitación (creado a mediados de los años de 1980) tendrá un rol importante en la organización de los entrenamientos que se dinamizan a través de la estrategia empresarial. La imposición a negociar el sistema de trabajo por turnos continuos reconoce un lugar destacado en los materiales empleados para capacitar a los mandos:

La industria automotriz está marcando nuevas pautas laborales de las cuales no podemos estar ajenos. Entre otras, General Motors, Fiat, Toyota y Chrysler, han acordado un régimen de trabajo flexible sobre la base de flexibilidad de horarios, polivalencias, refrigerio, remuneraciones, horas extras, vacaciones, productividad, etc. Los demás proveedores y terminales exigen el mismo tratamiento, de lo contrario quedaremos en desventaja.

¿Qué necesitamos? 1) Reducir el costo laboral en un 25\%;2) trabajar los 7 días de la semana; 3) Turnos rotativos; 4) Fraccionamiento programado de vacaciones durante todo el año; 5) Implementación automática de estándares de trabajo basados en 7 $1 / 2$ horas a un ritmo de $3 \frac{1}{2}$ millas por hora.

¿Qué podemos hacer? Aumentar la productividad de toda la planta en un 15\%; efectuar el pago de los bonos de producción, productividad y calidad en vales alimentarios; trabajar los 7 días de la semana para mejorar la distribución del costo fijo atenuando su incidencia sobre la producción realizada. Instaurar el sistema de "trabajo en equipo" eximiendo a la empresa del pago de la bonificación del 200\%, quedando en pie de igualdad con nuestros competidores inmediatos.

¿Cómo obtenemos el resto? Existe un programa de mejora continua de productividad que se orienta a recuperar los tiempos perdidos de los equipos, aprovechar el máximo rendimiento de los mismos e introducir mejoras en el diseño.

¿De qué forma vamos a alcanzar estas coincidencias? Negociando con el SUTNA eficientemente en el menor plazo posible. Si es necesario, como lo adelantara nuestro presidente en el 80 aniversario, trabajando 24 horas por día hasta alcanzar el objetivo (Material usado en los entrenamientos, empresa del sector del neumático localizada en Hurlingham).

Turnos y células asociados construyen la idea de un nuevo trabajador; en los primeros gobierna la intensificación y la alteración de la vida, en las segundas, el lenguaje de la valoración de la pericia técnica, de la innovación y de la participación. En cualquiera de los casos la mejora continua regla los estándares a ser alcanzados y superados.

\subsection{El Sistema de Producción Toyota (SPT): mejora continua en la intensificación del trabajo}

El programa de mejora continua de la productividad resulta en ese período y posteriormente un instrumento clave para el funcionamiento de las empresas. Sin embargo, el mayor desafío, que por cierto las empresas del neumático invocaban, era propiciarlo a través de la negociación. Cabe destacar que a medida que transcurren las décadas del 2000 y 2010, las cláusulas relacionadas con la doctrina gerencial procedente de Oriente se hacen cada vez más presentes en los convenios y actas. A mediados de los años de 1990 aún se trataba de un impulso 
que en el caso del sector del neumático conllevaba un conflicto laboral de importancia. En contraposición a esta situación, la empresa Toyota, radicada en ese período en Argentina, regula un primer convenio colectivo de trabajo donde, como se analiza más adelante, la filosofía de la mejora continua y con ella la entidad de los grupos kaizen cobran protagonismo en las regulaciones laborales.

En la terminal automotriz, instalada a mediados de los años de 1990, estos grupos se impulsaron especialmente como modalidad fundamental que da fisonomía a la organización del trabajo. A su vez, la flexibilidad para la mejora continua se reguló colectivamente. El denominado Sistema de Producción Toyota (SPT) se constituyó en el principio vertebral y direccionador de las doctrinas corporativas que se implementaron en varias empresas.

El complejo automotor ha sido un campo de problematización fundamental en los estudios sociales del trabajo en Argentina. Algunos aportes se inscriben en el análisis de los saberes requeridos en función de las normas de competitividad imperantes (Novick 2002; Sano y Martino 2003), en las lógicas sindicales, la negociación colectiva y la conflictividad laboral (Guevara 2010; Wyczykier 2011) y en los procesos identitarios, cuestión especialmente relevante al problematizar el modelo corporativo de Toyota y las exigencias empresarias para los jóvenes técnicos (Battistini 2001; Hernández y Busto 2009; Álvarez 2012). Nuestras investigaciones en terminales automotrices se han focalizado en las doctrinas corporativas y en los dispositivos de control laboral que se derivan de ellos. En ese contexto se profundizaron aspectos sensibles asociados con la matriz disciplinadora y su expresión en la gestión por competencias, el funcionamiento de los grupos de trabajo y la estrategia de formación corporativa.

En la empresa automotriz analizada, los operarios son jóvenes técnicos y realizan en la línea un trabajo intensivo que se entremezcla con las exigencias sistemáticas y permanentes de la mejora continua. El ritmo de la línea es incesante, la apuesta empresarial por reducir cada vez más los tiempos que demanda cada operación, supondrá también para los operarios un trabajo repetitivo. En ese marco, tienen lugar las exigencias corporativas derivadas de la mejora continua y el aporte requerido o exigido en tiempo real del saber obrero al servicio de la modificación de procesos que inciden favorablemente en la productividad.

El ritmo vertiginoso de la línea asociado con el trabajo simplificado cobra expresión en el marco de una entrevista grupal realizada con jóvenes técnicos (operarios):

\footnotetext{
Yo ahí estoy, en esa parte, debe hacer dos años y ya están pensando en moverme para otro lugar, como siempre ando dolorido de la cintura (...). Porque si vos estás en línea estás todo el día doblado (...) cuando yo entré estaba en diez minutos la línea, ahora dan tres. Vos decís diez minutos, me sobraba más y no, no me sobraba más (...) Bajan el tiempo de la línea para que saquen más cantidad de camionetas según el pedido de afuera (...) ya por inercia ni pensás lo que estás haciendo (operarios polivalentes de la filial argentina de la empresa Toyota).
}

El esfuerzo empresarial por reducir constantemente el tiempo de cada operación se expresa en el deterioro también constante de los trabajadores, sometidos a ritmos extenuantes y a una disciplina férrea. 
A la intensificación del trabajo se le suma paradójicamente la exigencia corporativa de aportar continuamente sugerencias para mejorar el proceso. Se instrumentan instancias institucionalizadas para movilizar el "aporte de sugerencias", que será tomado en cuenta en las evaluaciones y definirá las promociones. Es decir, en la intensificación, la colaboración:

\begin{abstract}
Si yo hago una mejora (kaizen) para tener otra categoría, pasa que sacan un provecho enorme, a lo mejor, le hiciste ahorrar un millón de pesos y a vos te pagan seis, siete pesos (...) o un viaje a Brasil a ver la Fórmula 1, en el día vas y venís. Y la empresa ahorró un millón de pesos por mes (operario polivalente).
\end{abstract}

Si la intensificación en la línea demanda saberes simplificados, los kaizen necesitan dinamizar, detrás de la búsqueda sistemática de innovación, saberes más complejos. La presión hacia los jóvenes se expresa permanentemente en ambas situaciones (Hernández 2006). En la terminal automotriz se articulan certeramente varios instrumentos para vehiculizar esta lógica de dominio sobre el trabajo humano: la negociación colectiva que regula el SPT, el funcionamiento de las células en el corazón del proceso de trabajo y la gestión de las competencias corporativas. El aporte de saberes no valorizados y el incesante ritmo de la línea son claramente observados por algunos técnicos, aunque distan de una estrategia de resistencia colectiva y de un accionar gremial que dispute con el accionar corporativo empresarial.

Los principios normativos del SPT fueron regulados en el primer convenio colectivo de trabajo que también presenta el sello del origen, ya que data de mediados de los años de 1990, cuando se instala la filial de la automotriz en Argentina. Ese convenio -firmado entre TASA y SMATA 190/96-, establecía en el artículo 1 (Filosofía de trabajo, declaración inicial) que

\footnotetext{
Ambas partes específicamente reconocen las especiales características del Sistema de Producción Toyota (SPT) (...) es indispensable una estructura de producción que privilegie la adaptabilidad laboral y la polivalencia en las funciones, para lograr el mejor auto al más bajo costo para el mayor número de clientes(Convenio Colectivo de Trabajo 190/96, art.1).
}

La referencia anterior es elocuente, en términos de la aceptación por parte del sindicato del SPT. Así, se expresa un reconocimiento que se constituye en piedra angular, sobre la que se edifica cualquier negociación, siempre y cuando responda a la filosofía Toyota.

A diferencia de otras empresas, el SPT unifica, de alguna forma, el orden normativo legal con el funcionamiento productivo-técnico. De allí, la ejemplaridad de un sistema que acopla los principios doctrinales en la lógica tecno-productiva. Desde el inicio de las actividades el Sistema de Producción Toyota constituirá doctrina y de ella derivarán complejos dispositivos que distribuirán eficazmente esos principios en los diferentes espacios de trabajo. El efecto disciplinador se juega en cada uno de los espacios y grupos de trabajo y así asume importantes implicancias en el "modelaje" del sujeto trabajador. La colaboración en la intensificación deja al descubierto aristas sensibles de las lógicas gerenciales contemporáneas, marco en el cual el aporte de los saberes productivos obreros se 
realiza a partir de un aparato disciplinador que se actualiza mediante el proceso técnico de trabajo y en las regulaciones laborales.

\subsection{Saberes técnicos en disputa: apropiación empresarial y resistencias}

La extracción de los saberes productivos obreros sigue constituyendo un instrumento de dominio del capital sobre el trabajo en la fase actual del capitalismo, hoy como derivación de la matriz toyotista. Así, se implementan variados dispositivos, agencias y agentes que asumen un papel clave en las normalizaciones y sistematizaciones del saber obrero. Y, esta estrategia, desplegada en contextos situados, gobierna y propicia una tensión permanente entre las competencias requeridas (con base en cierta tecnicidad relacionada directamente con los mandatos corporativos) por una parte y, por otra, el saber hacer y la experiencia acumulada en las trayectorias profesionales, culturales y de participación política de los trabajadores. El normal funcionamiento de las organizaciones se sustenta en sistemas corporativos transpuestos en el cotidiano laboral, de los que derivan exigencias corporativas específicas para los trabajadores. Pero también, la empresa sigue requiriendo el aporte del saber hacer obrero, de su pericia, de los saberes implícitos que movilizan, circulan, se recrean, transmiten en los espacios de trabajo. Incluso, y más allá que la línea de producción exija movilizar saberes simplificados, la dinámica corporativa configura condiciones para la movilización y apropiación de la tecnicidad obrera. El toyotismo ha instalado un sistema de captura en tiempo real a partir de potentes dispositivos (los grupos de trabajo) que posibilitan apropiarse de aquello que se crea y recrea en el espacio de trabajo, fruto de la experiencia acumulada. Nuestras investigaciones indican que esta captura sistemática se configura en un escenario propicio para impulsar cierta resistencia por parte de los trabajadores, quienes paradójicamente movilizan la creatividad del oficio y a la vez expresan disconformidad por su escasa valorización.

Durante los años de 1990 en Argentina, el contexto flexibilizador de desempleo estructural y creciente precarización implicó un vaciamiento del saber y experiencia acumulada. Es más, según estudios realizados en empresas privatizadas la nueva composición de la fuerza de trabajo implicó que se recontratarán trabajadores con una importante trayectoria y que habían sido desplazados de sus puestos de trabajo. En ese marco, el conocimiento técnico sistematizado fue expoliado, no sin severas consecuencias para el propio funcionamiento de las empresas.

Justamente, y en el contexto que se viene describiendo, en la empresa estudiada del sector neumático se pudo constatar cómo el saber hacer acumulado ponía claros límites para expandir generalizadamente la nueva organización del tiempo de trabajo, basada en el trabajo por turnos continuo. Esta situación se puso en evidencia, por ejemplo, en el sector Dúplex que requería calificaciones complejas y la puesta en juego del saber hacer fruto de la experiencia. La incorporación de personal contratado para cubrir un cuarto turno no se resolvía tan fácilmente en la práctica, ya que el nuevo personal no podía ser formado en tiempos breves. No obstante, esta situación será compensada por la labor 
desarrollada por los propios trabajadores en algunos sectores. Es decir, el ingreso de personal más joven para cubrir el cuarto turno, que demandaba la nueva organización, movilizaba también una estrategia empresarial destinada a que los más experimentados enseñen a los recién incorporados. Así se expresaba un delegado sindical sobre la cuestión planteada:

\begin{abstract}
El 6 (días de trabajo) X 2 (días de descanso) se firmó para toda la planta, pero en algunos sectores, como en Dúplex, no está implementado. Dúplex es el corazón de la planta donde se procesan los costados y las bandas de las cubiertas, donde salen los rodados, si no hay rodados no se puede construir. A ellos les falta un turno, se necesitarían 14 o 16 muchachos más para que se pueda hacer el 6 X 2, sino, cuando se toman el día de descanso tenés un día todo parado (...) no se puede preparar un trabajador para Dúplex de un día para otro, por eso es que van tomando y van practicando hasta tener un plantel (...) es un departamento atípico, porque la gente tiene que tener condiciones especiales, donde se construye, hay que tener en cuenta el calibre, el peso, la calidad de la goma. Es el sector que requiere más calificación (Dirigente sindical, Sindicato Único de Trabajadores de la Actividad del Neumático).
\end{abstract}

Según la investigación realizada, la función formadora, que cobra centralidad en el marco de la gerencia de recursos humanos, se descentraliza en los diferentes sectores de trabajo. Si bien se pone en evidencia su jerarquización a través de una institucionalización de la función formadora, la efectividad se expresa en tanto y en cuanto se transponga eficazmente e involucre a todos los trabajadores, aunque con funciones diferenciadas. Es decir, el orden corporativo conlleva un nuevo orden pedagógico que posibilita la transposición de los principios de legitimidad imperantes. Las funciones formadoras de inducción (en los reclutamientos, por ejemplo, de inmersión en las doctrinas corporativas), el aprendizaje de las reglas definidas del buen desempeño y el involucramiento requieren, sin embargo, entramarse con la experiencia movilizada en el acto de trabajo a través de la pericia técnica. Justamente, allí radica la cooperación, y es justamente por esto que las herramientas corporativas asociadas con los programas de mejora continua tienden a capturar esta pericia. En ese contexto, se dinamiza una vigilancia estratégica a cargo de los mandos: con relación a la transmisión de saberes productivos, a su movilización y a la efectiva circulación de los saberes oficiales empresariales (las competencias corporativas). A este proceso se denomina la pedagogización del espacio de trabajo. Si bien no se trata de una novedad en la historia del capitalismo, sí adquiere una forma concreta a la luz de las doctrinas corporativas contemporáneas.

En las últimas dos décadas los dispositivos pedagógicos empresariales han cobrado protagonismo, así como su relación estrecha con la gestión de las competencias laborales. Formación y competencias se traducen en sofisticados sistemas de clasificación que se derivan, en diversas empresas, de los propios sistemas corporativos. Los horizontes profesionales se hacen mucho más difusos, limitados y las posibilidades de movilidad se resienten sensiblemente. El buen desempeño medido anualmente por los jefes define a veces la movilidad ascendente.

En la terminal automotriz estudiada, el orden corporativo y pedagógico establece un vínculo orgánico con el orden técnico-productivo y normativo que 
se regula colectivamente. Las exigencias de la empresa hacia los trabajadores de propiciar mejoras en los procesos se vive como una carga importante en el día a día. Además, estas mejoras son reconocidas a los efectos de gestionar las promociones:

\footnotetext{
Él (en referencia a un operario) modificó una parada de línea, un sensor de la línea (saca) ruedas, cada tanto se venía parando. Entonces en el día si vos lo sumas, por día paraba quince minutos, eso lleva un cálculo de plata que se pierde estando parada. Entonces hizo un cálculo y al año le daba más o menos un millón de pesos que ahorraba la empresa con esta mejora. Se lo aceptaron, fue recontra bueno y le dieron un viaje (Operario polivalente en la línea de producción).
}

En los distintos sectores de trabajo el saber obrero, a través de los kaizen, se pone a disposición de la empresa; este aporte, que paradójicamente se realiza en un contexto de exigencias de saberes simplificados, alcanza un límite: llega un momento en que la rotación tiene lugar. No se trata solo de lo insoportable que resulta la línea, también la rotación posibilita para la empresa nuevas contribuciones obreras para la mejora de procedimientos al incorporarse los operarios a nuevos sectores de trabajo. Al respecto un operario comenta: "Por ejemplo, el chico que está en ensamble y labura en una operación de tres metros y medio para recorrer y llega un momento ¿qué vas a mejorar? Le pusiste un tarrito a la pistola para acomodarla, después pusiste un cartelito y llega un momento ¿qué más vas a poner?" (Operario en la línea de producción). Otro operario también plantea: "Y ahí es donde viene la rotación. Traen otro nuevo que vea algo distinto, muy hábil. (...) Claro, por eso el recambio" (Operario polivalente en la línea de producción).

La consolidación de la hegemonía empresarial se sustenta en el afinamiento de varias herramientas que posibilitan una mejor articulación entre los saberes corporativos (sustento de las doctrinas corporativas y viabilizados por la gestión del conocimiento oficial empresarial) y los saberes técnicos que aportan los trabajadores. Justamente, consolidar el vínculo orgánico entre ellos, demanda afinar instrumentos de formación locales (en el nivel de los sectores de trabajo) y movilizar un sistema de evaluaciones de desempeño que va teniendo cada vez mayor importancia en la movilidad profesional. En este marco, la extracción y movilización de la tecnicidad obrera en los grupos de trabajo posibilita una mayor articulación entre saberes corporativos y saber hacer $\mathrm{y}$, de esta forma, coadyuva en el sostenimiento del orden empresarial.

\section{Exigencias corporativas y clasificaciones profesionales}

Las prácticas hegemónicas empresariales se expresan también sensiblemente en las condiciones de profesionalización de los operarios. Desde finales de los años de 1980, la instrumentación de nuevos criterios en el reclutamiento y la movilidad profesional se asocia con las doctrinas gerenciales contemporáneas de la mejora continua y, en ese marco, con la gestión por competencias. En la última década, esos criterios se van plasmando en las regulaciones laborales. En el 
caso de los trabajadores idóneos ${ }^{9}$ existen crecientes limitaciones para la movilidad profesional y para poder acceder a puestos de conducción, que antes era posible mediante la carrera interna - marco en el cual se valorizaba la experiencia acumulada y el saber hacer-. En la actualidad, las competencias, los liderazgos y las evaluaciones de desempeño son aspectos sensibles a la hora de resolver la movilidad, más que la pericia técnica obrera indispensable para el normal funcionamiento de las empresas (Spinosa y Drolas 2009; Bureau y Tuchszirer 2010; Kergoat 2012).

Algunas de las investigaciones en la década de 1990 ya indicaban cómo las nuevas categorías empezaban a investirse de un nuevo lenguaje: el operador en vez del operario y el facilitador, asesor o tutor en lugar del supervisor. Por ejemplo, en estudios que realicé en una empresa textil-química y farmacéutica, en el segundo lustro de los años de 1990, pudimos constatar que la potencial movilidad entre categorías se sustentaba en la valorización de una experiencia que debía demostrar la fusión con el "perfil de empresa"

En la industria del neumático, la invocación recurrente al "necesario cambio cultural" se expresa especialmente en los supervisores, quienes se denominarán facilitadores. La semántica de la mejora continua se entremezcla con la doctrina flexibilizadora al regular el trabajo por turnos continuo (Drolas 2010). Todavía en la década de 1990 y más allá de la persistente invocación al cambio cultural y a las nuevas figuras de facilitadores y operarios líderes, las categorías profesionales eran las reguladas en el Convenio Colectivo de Actividad núm. 101 de 1975 (firmado entre el SUTNA y empresas de la actividad del neumático).

La transformación del orden clasificatorio que se venía implementando en los hechos, como derivación de la consolidación de los sistemas corporativos, será en la última década traducida en forma creciente en las regulaciones laborales" ${ }^{11}$ La transmisión de la cultura empresarial por la mejora continua se va transparentando en un orden legal-normativo (expresado en los reglamentos de fábrica y en los CCT).

En la década de 1990, la flexibilidad regulada por la norma estatal y a través de la negociación colectiva fue previamente instalada en las empresas de hecho. De igual forma, las herramientas corporativas articuladas en torno al principio de la mejora continua -competencias, evaluaciones de desempeño, trabajo en células, etc. - fueron promovidas en el espacio de las empresas y tendrán cada vez mayor presencia en las regulaciones laborales negociadas a medida que transcurren los años del $2000^{12}$. También, los sentidos flexibilizadores siguen teniendo presencia en las actas de acuerdo y convenios colectivos de trabajo.

9 Aquellos trabajadores que han adquirido la pericia del oficio a través de una extensa trayectoria, pero no cuentan con la credencial educativa de nivel medio técnico.

10 El operario no era considerado parte de la carrera de operador, no obstante su labor resultaba indispensable para demostrar el "potencial" para transitarla. Cabe destacar que las bandas salariales para el operario y para el operador se diferenciaban; la movilidad para el puesto de facilitador se posibilitaba a partir de ascender a operador (Figari 2004).

11 Los convenios negociados en la industria del neumático en el último lustro de la década del 2000 (CCT 486/2007 y CCT 636/2011 entre SUTNA y las empresas FATE, Pirelli y Firestone) incorporan herramientas corporativas claves relacionadas con los principios toyotistas, como los grupos kaizen. También se regula el trabajo por turnos continuo.

12 Más allá de la negociación del trabajo por turnos y de la implementación de la mejora continua, hacia finales de los años de 1990 se cerró la planta radicada en Hurlingham, Gran Buenos Aires (con casa matriz en EE.UU.), lo que significó la pérdida de casi mil puestos de trabajo. 
El caso de Toyota es emblemático. La doctrina corporativa reconoce un anclaje fundamental: las células o grupos de trabajo y su relevancia queda expresada en la propia determinación de las categorías profesionales: operario polivalente y operario polivalente líder de célula o team leader. La descripción de funciones del operario polivalente supone, en primer lugar, "conocimiento de técnicas del sistema de producción Toyota" (CCT 190/96 E, art. 16) $)^{\text {13; }}$ la polivalencia es caracterizada a partir de un desempeño que puede desenvolverse en diferentes sectores (ensamblaje, pintura, soldadura, tapicería, etc.), pero también a partir de funciones técnicas que integran el control, la preparación de herramientas y máquinas, el mantenimiento, el orden y la limpieza. El team leader tendrá las mismas funciones, sumándole aquellas relacionadas con la asistencia y la capacitación -en el puesto o tarea-. En la modificación del CCT -en el 2009-, se amplían las funciones del team leader que abarcan la capacitación, claramente asociada a una función de control y evaluación de los operarios polivalentes:

\section{(...) deberá ser apto para capacitar a los operarios de la categoría precedente en to- das y cada una de las funciones y tareas de la célula. (...) Colaborará activamente en la difusión, aplicación y control del TPS (Jidoka, just-in-time, trabajo estandarizado y kaizen) asistiendo en la detección y corrección de desvíos. (...) Concientizará al operario polivalente sobre la importancia de la concurrencia diaria al trabajo y con- tribuirá a fomentar e incentivar el trabajo en equipo, reportando situaciones anó- malas (...) (CCT 730/2005 y su modificatoria, con Resolución Nacional 12/2009).}

La difusión, aplicación y control de la doctrina corporativa exige una transmisión eficaz (hacia los grupos y hacia el operario polivalente) que busca avanzar incluso hacia la concientización. En la modificación del convenio (en el 2009) se introduce el criterio de antigüedad y su valorización en términos salariales. Así, se estaría expresando cierto reconocimiento de la experiencia corporativa que debió ser demostrada en las recurrentes evaluaciones de desempeño y habilidades. Estas evaluaciones se incorporan en la modificación al CCT en el 2005 y se ajustan asimismo en el 2009 al regular aspectos relacionados con la difusión de los objetivos que se tienen que alcanzar y la comunicación a cada evaluado (Art. 21: Evaluación de desempeño y habilidades; Art. 35.3: Antigüedad). De esta for$\mathrm{ma}$, las clasificaciones reguladas expresan en esta empresa un vínculo orgánico con la doctrina corporativa. Como sostén de dicho vínculo, cobra importancia la función formadora en los espacios de trabajo que se constituye en una vía potente de encauzamiento de los principios corporativos. Las posibilidades de movilidad son acotadas y dependerán del "buen comportamiento laboral", según lo requiere el SPT.

\section{Conclusiones}

La consolidación de la hegemonía empresarial ha requerido en las últimas dos décadas articular, especializar y transponer eficazmente las reglas corporativas en el cotidiano laboral. Los operarios que se desempeñan en la línea de producción directa son blanco de las persistentes manifestaciones de la doctrina

13 Esta regulación se mantiene en el CCT 730/2005. 
flexibilizadora articulada con las lógicas modernizantes derivadas de la impronta toyotista de la mejora continua. Las tradiciones investigativas desarrolladas desde la década de 1990 han sido fértiles a los efectos de identificar las tendencias predominantes, algunas de ellas prefiguradas en esa década y consolidadas en la primera del milenio. Los dos casos analizados en este artículo aportan hallazgos contundentes con relación a las persistentes formas de intensificación del trabajo humano y su vehiculización a través de la negociación colectiva, ya sea a partir de actas de acuerdo, como en el caso del subsector de la industria del neumático, o bien por medio de convenios colectivos de trabajo (como en el caso de la terminal automotriz). Asimismo, las lógicas modernizantes de la mejora continua generan exigencias corporativas que buscan paradójicamente la colaboración, el buen desempeño y el aporte de mejoras en los procesos a través de la disponibilidad continua de la pericia técnica obrera indispensable para el normal funcionamiento de las firmas. La praxis empresarial se especializa en los efectos de transponer eficazmente en los espacios de trabajo la doctrina gerencial sustentada en la mejora continua. Este proceso exige articular las competencias corporativas con los requerimientos técnicos y productivos de cada sector de trabajo. La organización del trabajo basada en el trabajo en grupos en general, y en los kaizen en particular posibilita: la interpenetración de las competencias corporativas y técnicas; la apropiación empresarial en tiempo real de los saberes productivos; la producción de nuevos saberes por parte de los trabajadores que permanentemente aportan mejoras en los procesos al movilizar su actividad cognitiva y los saberes implícitos de difícil codificación. La apropiación de la pericia técnica nutre las doctrinas corporativas y facilita una adaptación según las exigencias productivas. En este contexto se institucionaliza un aparato de formación que se deslocaliza en los sectores de trabajo para luego enriquecer y consolidar la doctrina corporativa. En este proceso los mandos cobran protagonismo. Así, se establece un vínculo orgánico entre el orden corporativo y pedagógico lo que posibilita la consolidación de la hegemonía empresarial.

Resulta relevante destacar que las resistencias obreras se expresan en relación tanto con la intensificación del trabajo como a partir de la apropiación de la pericia obrera posibilitada por la aplicación de herramientas corporativas asociadas con la mejora continua. Para funcionar, la empresa requiere del saber hacer obrero, justamente, el desafío es cómo generar instancias institucionalizadas $-\mathrm{y}$ descentralizadas, a la vez- de captura (del saber técnico) en tiempo real. Las clasificaciones, reguladas o de hecho, constituyen una vía para sostener la cooperación sobre la base de la "distinción" de algunos trabajadores; de esta forma se recrean colectivos basados en la competencia como motor de eficiencia. Las clasificaciones traducen, a la vez, más tarea y más colaboración como factores que se ponderan en los ascensos. En el contexto descrito, el orden profesional se consolida en relación estrecha con la matriz corporativa de control social y cultural que se impulsa hacia los trabajadores.

En consecuencia, el examen detenido de los saberes en disputa -inscribiéndolo en el conflicto material- aporta elementos de interés para analizar los mecanismos operantes en la matriz de control que se consolida en estrecha vinculación con las competencias sociales y reguladoras, y la captura continua de la pericia técnica obrera. El aporte obrero no es traducido necesariamente en un 
debate relacionado con las clasificaciones profesionales, lo que deriva en una escasa incidencia en los sistemas de jerarquización salarial. Lo que está en disputa también es la consolidación de configuraciones profesionales con base en las matrices corporativas sustentadas en una doctrina flexibilizadora persistente y en los principios unificantes de la mejora continua en el capitalismo global.

\section{Referencias bibliográficas}

Alonso, Luis y Carlos Fernández. «La innovación social y el nuevo discurso del management: limitaciones y alternativas». Arbor, Vol. 187, n 752, 2011: 97-118.

Alonso, Luis y Carlos Fernández. «Los discursos del management. Una perspectiva crítica». Lan Harremanak. Revista de Relaciones Laborales, $n^{\circ}$ 28, 2013: 42-69.

Álvarez, Diego. «Organización del trabajo y dispositivos de control en el sector automotriz: el toyotismo como sistema complejo de racionalización». Trabajo y Sociedad, $\mathrm{n}^{\circ}$ 18, 2012: 43-57.

Alves, Giovanni. "Crise estrutural do capital, trabalho imaterial e modelo de competência: notas dialéticas». En Trabalho e educação. Contradições do capitalismo global, organizado por Giovanni Alves, Jorge Gonzalez y Roberto Batista, 47-81. Brasil: Projeto Editorial Praxis, 2006.

Angélico, Héctor y Claudia Figari. 1999. La organización del tiempo de trabajo en la estrategia de flexibilización: laboral estudio de caso en la industria del neumático. Ponencia presentada en el "IV Simposio Nacional de Análisis Organizacional", II del Cono Sur, Centro de Estudios Organizacionales de la Facultad de Ciencias Económicas de la UBA. Buenos Aires, 11 al 13 de agosto.

Antunes, Ricardo. "O trabalho, sua nova morfologia e a era da precarização estrutural». Revista Theomai, Estudios sobre Sociedad y Desarrollo, n 19 (primer semestre 2009): 47-57.

Batista, Roberto. "A panaceia das competências: uma problematização». En Trabalho e educação. Contradições do capitalismo global, organizado por Giovanni Alves, Jorge Gonzalez y Roberto Batista, 82-114. Brasil: Projeto Editorial Praxis, 2006.

Battistini, Osvaldo. «Toyotismo y representación sindical. Dos culturas dentro de la misma contradicción». Revista Venezolana de Gerencia, n 16, 2001: 533-572.

Bernstein, Basil. Pedagogía, control simbólico e identidad. Madrid: Morata, 1998.

Bourdieu, Pierre. La distinción. Criterio y bases sociales del gusto. Madrid: Taurus, 1984.

Bureau, Marie-Christine y Carole Tuchszirer. «La validation des acquis de l'expérience est-elle un moyen de reconnaissance du travail?» Sociologie du travail, $\mathrm{n}^{\circ}$ 52, 2010: 55-70.

Datchary, Caroline. «Gérer la dispersion: un travail collectif». Sociologie du travail, $\mathrm{n}^{\circ}$ 50, 2008: 396-416.

Delfini, Marcelo. «Relaciones laborales y "gestión de recursos humanos" en filiales de empresas multinacionales en Argentina». Sociedad y economía, $\mathrm{n}^{\circ} \mathbf{2 0}$, 2011: 171-195.

Delgado, Eric. «La responsabilidad social empresaria y los valores cooperativos. Dos cuestiones distintas». Realidad Económica, n² 230, 2007: 47-60. 
Dombois, Rainer. «Modernización industrial, reto para las relaciones industriales en América Latina». Revista Colombiana de Psicología, n 3, 1994: 81-92.

Drolas, Ana. «Los sindicatos frente a la política de competencias. Aportes para un debate necesario». Revista Theomai, $\mathrm{n}^{\circ} 21$ (primer semestre 2010): 62-75.

Edwards, Paul y Margaret Collinson. «Empowerment and Managerial Labor Strategies: Pragmatism Regained». Work and Occupations, Vol. 29, n 3, 2002: 272-299.

Elias, Norbert. El proceso de la civilización. Investigaciones sociogenéticas y psicogenéticas. Buenos Aires: Fondo de Cultura Económica, 1993.

Figari, Claudia. 2004. Saberes, sujetos y posiciones en el nuevo orden empresario: Dispositivos de control y configuraciones profesionales emergentes. Tesis doctoral con orientación en Ciencias de la Educación, Universidad de Buenos Aires.

Figari, Claudia. «Lógicas de formación, gestión por competencias y nuevas configuraciones profesionales». En Trabajo y formación en debate: saberes, itinerarios y trayectorias de profesionalización, compilado por Claudia Figari, Martin Spinosa y Julio Testa, 21-42. Buenos Aires: Ciccus, $2011 a$.

Figari, Claudia. "Work Discipline and Corporate Training in Modernising Large Companies in Argentina». Work Organisation, Labour and Globalisation, Vol. 5, $\mathrm{n}^{\circ}$ 1, 2011b: 130-149.

Figari, Claudia. «Hegemonía empresarial y mediaciones pedagógicas en los espacios de trabajo». Revista Sociología del Trabajo, $\mathrm{n}^{\circ} 78$ (segundo cuatrimestre 2013): 95-115.

Friedmann, Georges y Pierre Naville. Tratado de sociología del trabajo. México D.F.: Fondo de Cultura Económica, 1971.

Giniger, Nuria. «El ojo del amo engorda al ganado: estrategias de control y disciplinamiento de la fuerza laboral». Trabajo y Sociedad, Vol. XV, $\mathrm{n}^{\circ} 16$ (verano 2011): 125-135.

Giniger, Nuria. «Nuevos paradigmas en las praxis empresariales. Transiciones en los cambios de propiedad de una empresa siderúrgica, en Argentina». Revista Enfoques, Edição "Mundos do Trabalho: novos contextos, novas perspectivas", Vol. 11, $n^{\circ}$ 1, 2012: 160-177.

Graciolli, Edilson y Paulo Lamana. «Responsabilidade social empresarial e Estado neoliberal». En Trabalho, Educação e sociabilidade, editado por Jose Dos Santos y Renan Araujo, 115-129. Brasil: Projeto Editorial Praxis, 2010.

Guevara, Sebastián. «La acción gremial de los trabajadores automotrices [sic] en Argentina, desde la posdevaluación hasta la crisis actual». Perfiles Latinoamericanos, $\mathrm{n}^{\circ}$ 36, 2010: 121-141.

Hernández, Marcelo. 2006. Obreros jóvenes y con estudios secundarios completos: algo más que una excentricidad del capital. Estudio de caso en una fábrica de la industria automotriz. Ponencia presentada en el "I Congreso multidisciplinario de ciencias sociales: 'La exclusión y los nuevos paradigmas de la complejidad social'", Mérida, Venezuela, Universidad de Los Andes, marzo.

Hernández, Marcelo y Cristian Busto. «Organización de la producción, imposición de sentidos corporativos y resistencias: el caso de una empresa automotriz». En La precarización del trabajo en América Latina. Perspectivas del 
capitalismo global, editado por Claudia Figari y Giovanni Alves, 203-230. Brasil: Projeto Editorial Praxis, 2009.

Jonnaert, Philippe, Johanne Barrette, Domenico Masciotra y Mane Yaya. «La competencia como organizadora de los programas de formación: hacia un desempeño competente». Revista de Currículum y Formación de Profesorado, $\mathrm{n}^{\circ}$ 12, 2008: 1-32.

Kergoat, Prisca. «Pensar a cultura operária para desconstruir a hierarquização dos saberes». Educação \& Sociedade, Vol. 33, n 118, 2012: 47-59.

Kuenzer, Acácia. Pedagogia da fábrica. As relações de produção e a educação do trabalhador. São Paulo: Cortez Editora, 2011.

León, Francisco. «La lógica de los trabajadores. Un estudio sobre la racionalidad, la autonomía y la coherencia de las prácticas y los significados de los trabajadores». Revista Internacional de Sociología (RIS), Vol. 67, $\mathrm{n}^{\circ}$ 1, 2009: 135-160.

Lichtenberger, Yves. Competencias y calificación. Cambios de enfoques sobre el trabajo y nuevos contenidos de negociación. Buenos Aires: Piette, Conicet, 2000.

Lima, Jacob. «Participação, empreendedorismo e autogestão: uma nova cultura do trabalho?» Sociologías, Año 12, n² 25, 2010: 158-198.

Linhart, Danièle. La modernización en las empresas. Buenos Aires: Asociación Trabajo y Sociedad, 1997.

Lukács, Georg. Historia y conciencia de clase. Madrid: Sarpe, 1985.

Marshall, Adriana y Laura Perelman. «Cambios en los patrones de negociación colectiva en Argentina». Estudios Sociológicos, Vol. XXII, n 65, 2004: 409-434.

Mészáros, István. O poder da ideologia. Sao Paulo: Boitempo, 2004.

Monchatre, Sylvie. "Gestión por competencias y relación salarial». Trayectorias, Vol. 11, n² 28, 2009: 102-117.

Montes, Juan. «La configuración del poder en los espacios de trabajo: dispositivos disciplinarios y resistencia de los trabajadores». Revista de Sociología del Trabajo, $\mathrm{n}^{\circ}$ 54, 2005: 73-99.

Muñiz, Leticia. «Trayectorias laborales fragmentadas en un contexto de privatización: un estudio de caso en Argentina». Sociología del trabajo, n 65, 2009: $53-80$.

Neves, Lucia (Org.). A nova pedagogia da hegemonia. Estratégias do capital para educar o consenso. Sao Pablo: Xamã Editora e Gráfica Limitada, 2005.

Novick, Marta. "Aprendizaje y conocimiento como ejes de la competitividad capacitación e innovación en dos tramas productivas de la industria manufacturera argentina». En Sistemas locales y capacitación, editado por María Ibarrola, 103-135. Montevideo: Cinterfor-OIT, Universidad de León, RET, 2002.

Ollivier, Carine. «Carrière du capital social et segmentation du marché: évolution des effets des relations sur les trajectoires professionnelles des architectes d'intérieur». Sociologie du travail, $\mathrm{n}^{\circ}$ 53, 2011: 52-74.

Quintana, Francisco. «Actividad sociocognitiva y contextos postfordistas». Revista Internacional de Sociología (RIS), Vol. 67, n 2, 2009: 347-371.

Riquelme, Graciela y Natalia Herder. 2007. Saberes en jaque: de la negociación de las calificaciones a la certificación de las competencias. Ponencia presentada en el "V Congreso Latinoamericano de Sociología del Trabajo", ALAST, Montevideo, 18 al 20 de abril. 
Roper, Juliet, Shivy Ganesh y Kerr Inkson. «Neoliberalism and Knowledge, Interests in Boundaryless». Work, Employment, Society, Vol. 24, n 4, 2010: 661-679.

Rozemblatt, Patrick. El cuestionamiento del trabajo. Clasificaciones, jerarquía, poder. Buenos Aires: Piette, 1999.

Sano, Makoto y Luis Martino. «Tres casos de japonización de la relación de empleo en Argentina». Revista de la CEPAL, n 80, 2003: 185-195.

Soich, Darío. «Disciplina fabril y estrategias de dominación corporal en una corporación automotriz trasnacional». Revista Runa, $n^{\circ}$ 28, 2008: 93-110.

Spinosa, Martin y Ana Drolas. «De la certificación-formación, a la certificaciónaprendizaje en el proceso de integración del Mercosur. Calificaciones y empleo. Dimensiones francesas y europeas de la formación y el empleo». Piette / Cereq, $\mathrm{n}^{\circ}$ 62, 2009: 1-4.

Stewart, Paul, José Ramalho, Andy Danford, Valeria Pulignano y Marco Santana. «Novas estratégias gerenciais e a qualidade de vida no trabalho na indústria automobilística (Gra-Bretanha, Brasil e Itália)». Revista Latinoamericana de Estudios del Trabajo, Año 10, $\mathrm{n}^{\circ}$ 17, 2005: 165-188.

Tanguy, Lucie. «De la evaluación de los puestos a la de las cualidades de los trabajadores. Definiciones y usos de la noción de competencias». En El futuro del trabajo-el trabajo del futuro, compilado por Enrique de la Garza y Julio Neffa, 111-128. Buenos Aires: Clacso, 2003.

Wyczykier, Gabriela. «Constitución de identidades colectivas: los trabajadores y sus prácticas. Notas para pensar la acción gremial de base y la precariedad laboral en el sector industrial argentino». Trabajo y Sociedad, Vol. XV, $\mathrm{n}^{\circ} 17$ (invierno 2011): 285-308.

\section{Fuentes primarias}

\section{Entrevistas}

En la filial argentina de la empresa Goodyear del sector del neumático:

Dirigentes sindicales de la seccional del Sindicato de Empleados y Personal Jerárquico de la Actividad del Neumático Argentina (SEPJANA). Entrevistados por Héctor Angélico y Claudia Figari, marzo y mayo de 1997, en la ciudad de Buenos Aires y en la localidad de Hurlingham, Gran Buenos Aires, Argentina.

Dirigente sindical de la seccional Hurlingham, del Sindicato Único de la Actividad del Neumático (SUTNA). Entrevistados por Héctor Angélico y Claudia Figari, septiembre de 1997, en la localidad de Hurlingham, Gran Buenos Aires, Argentina.

Abogado laboralista interviniente en el proceso de negociación colectiva relacionado con la implementación del trabajo por turnos continuo. Entrevistado por Claudia Figari, mayo de 1998, ciudad de Buenos Aires, Argentina.

Talleres con trabajadores y delegados sindicales del SUTNA. Entrevista coordinada por Claudia Figari, agosto de 1997, en la localidad de Hurlingham, Gran Buenos Aires, Argentina.

\section{En la filial argentina de la empresa Toyota}

Entrevista grupal a técnicos que se desempeñaban en la línea de producción y en el sector de mantenimiento como operarios polivalentes. Entrevistados 
por Diego Álvarez Newman, Marcelo Hernández, Matías Frisco, Cecilia Rossi; bajo la coordinación de Claudia Figari, agosto de 2010, ciudad de Zárate, provincia de Buenos Aires, Argentina.

\section{Convenios colectivos de trabajo y actas de acuerdo}

\section{En el sector de la industria del neumático}

Actas acuerdo de productividad, en el sector Dúplex, 1992 (firmados entre la seccional Hurlingham del SUTNA y la empresa Goodyear).

Actas de acuerdo de productividad, 1994 (firmados entre la seccional San Fernando del SUTNA y la empresa Fate).

Actas acuerdo en el nivel de empresa que regulan el trabajo por turnos continuo, 1996 entre el SUTNA y la empresa Goodyear.

Actas acuerdo en el nivel de empresa que regulan el trabajo por turnos continuo, 1997 entre el SUTNA y la empresa Fate.

CCT de actividad, núm: 101, 1975 (firmado entre el SUTNA y empresas de la actividad del neumático).

CCT 486/2007 y CCT 636/201l (firmados entre el SUTNA y las empresas FATE, Pirelli y Firestone).

Material usado en los entrenamientos de trabajadores empresa Goodyear.

\section{En la filial argentina de la empresa Toyota}

CCT 190/96, CCT 449/2001 y CCT 730/2005 y su modificatoria, con Resolución Nacional 12/2009 (firmados entre el Sindicato de Mecánicos y Afines del Transporte Automotor -SMATA- y TASA).

Reportes de sustentabilidad (2008-2012), filial argentina empresa Toyota: www. toyoyanet.com.ar/18-reportes-de-sustentabilidad.note.aspx (último acceso: 30 de junio de 2014). 